\title{
Extraskeletal myxoid chondrosarcoma: tumor response to sunitinib
}

\author{
Silvia Stacchiotti ${ }^{*}$, Gian Paolo Dagrada ${ }^{2}$, Carlo Morosi ${ }^{3}$, Tiziana Negrii ${ }^{2}$, Antonella Romanini ${ }^{4}$, Silvana Pilotti ${ }^{2}$, \\ Alessandro Gronchi ${ }^{5}$ and Paolo G Casali ${ }^{1}$
}

\begin{abstract}
Background: Extraskeletal myxoid chondrosarcoma (EMCS) is a rare soft tissue sarcoma of uncertain differentiation, characterized in most cases by a translocation that results in the fusion protein EWSR1-CHN (the latter even called NRAA3 or TEC). EMCS is marked by $>40 \%$ incidence of metastases in spite of its indolent behaviour. It is generally resistant to conventional chemotherapy, and, to the best of our knowledge, no data have been reported to date about the activity of tirosin-kinase inhibitor (TKI) in this tumor. We report on two consecutive patients carrying an advanced EMCS treated with sunitinib.
\end{abstract}

Methods: Since July 2011, 2 patients with progressive pretreated metastatic EMCS (Patient1: woman, 58 years, PS1; Patient2: man, 63 years, PS1) have been treated with continuous SM 37.5 mg/day, on an individual use basis. Both patients are evaluable for response. In both cases diagnosis was confirmed by the presence of the typical EWSR1CHN translocation.

Results: Both patients are still on treatment (11 and 8 months). Patient 1 got a RECIST response after 4 months from starting sunitinib, together with a complete response by PET. An interval progression was observed after stopping sunitinib for toxicity (abscess around previous femoral fixation), but response was restored after restarting sunitinib. Patient 2 had an initial tumor disease stabilization detected by CT scan at 3 months. Sunitinib was increased to $50 \mathrm{mg} /$ day, with evidence of a dimensional response 3 months later.

Conclusions: Sunitinib showed antitumor activity in 2 patients with advanced EMCS. Further studies are needed to confirm these preliminary results.

Keywords: Sarcoma, Myxoid extraskeletal chondrosarcoma, Sunitinib malate, Targeted therapy, Chemotherapy

\section{Introduction}

Extraskeletal myxoid chondrosarcoma (EMCS) is a rare soft-tissue sarcoma (STS) first described in 1972 [1,2]. EMCS is now considered a unique entity of uncertain differentiation (convincing evidence of a cartilaginous differentiation is lacking in most cases) [3]. Besides, EMCS can have a neuroendocrine differentiation [4-6].

It is market by a specific chromosomal translocation, $\mathrm{t}(9 ; 22)(\mathrm{q} 22.3 ; \mathrm{q} 12.2)$, fusing $C H N$ to EWSR1 [7,8]. Less frequently two different translocations, $\mathrm{t}(9 ; 17)(\mathrm{q} 22.3 ; \mathrm{q} 12)$ and $\mathrm{t}(9 ; 15)(\mathrm{q} 22.3 ; \mathrm{q} 21.3)$ are found, resulting in RBP56$C H N$ and TCF12-CHN fusion-genes, respectively $[9,10]$.

\footnotetext{
* Correspondence: silvia.stacchiotti@istitutotumori.mi.it

'Department of Cancer Medicine, Adult Sarcoma Medical Oncology Unit, Fondazione IRCCS Istituto Nazionale Tumori Milan, via Venezian 1, 20133, Milan, Italy

Full list of author information is available at the end of the article
}

The fusion-proteins promote cellular growth and differentiation [11]. Furthermore, the EWSR1-CHN fusion-protein may activate the PPARG nuclear receptor gene [12]. Microscopically, EMCS can be subdivided into a conventional well-differentiated and a cellular high-grade EMCS, which is marked by epithelioid cells with prominent nucleoli, high mitotic rate and necrosis [13]. Dedifferentiated ECMS were also described [14].

Most EMCS arise from the deep soft tissues of the extremities and limb girdles [15-20]. The natural history is usually characterized by an indolent behavior, but studies with a long median follow-up show a high-rate of late local and distant tumor recurrence despite a prolonged clinical course [16-21]. Metastases are reported in around $40 \%$ of cases, with a $58 \%$ overall survival rate at 15 years in the largest retrospective series [20].

\section{Biomed Central}

(c) 2012 Stacchiotti et al.; licensee BioMed Central Ltd. This is an Open Access article distributed under the terms of the Creative Commons Attribution License (http://creativecommons.org/licenses/by/2.0), which permits unrestricted use, distribution, and reproduction in any medium, provided the original work is properly cited. 
Standard treatment of primary EMCS is complete surgical resection, followed by radiation in high-risk cases [21]. Patients with advanced disease usually receive a medical treatment. Unfortunately, response rates to conventional chemotherapy are low [15,21-24].

\section{Case report}

A 53-year female patient was diagnosed with a $20-\mathrm{cm}$ mass located to the right thigh in October 2006. Concomitant ipsilateral iliac lymph nodes (LN) were present. Both sites were biopsied, with a pathologic diagnosis of EMCS (Figure 1). In both samples histology showed a diffuse pancytokeratin (AE1/AE3) cytoplasmic staining and PPARG nuclear expression, whileS-100 protein, smooth muscle actin, synaptophysin and brachyury were negative. EWSR 1 and CHN gene status was investigated by dual-color break-apart FISH which detected a balanced rearrangement for both genes. Figure 1D shows the FISH rearranged pattern for $C H N$.

The identification of a $C H N$ translocation partner ruled-out the diagnosis of myoepithelial carcinoma, raised by morphology and cytokeratin immunoreactivity, as well as an ossifying fibromixoid tumor, another ECMS mimic [25]. Indeed, a subset of soft tissue myoepithelial carcinomas were recently reported to harbour EWSR1POU5F1 chimera [26] widening the growing family of
EWSR1 gene-rearranged tumors and thus making the EWSR1 rearrangement test alone unable to discriminate.

The patient was treated with anthracycline-based chemotherapy. This was followed by a wide excision of the primary tumor en-bloc with a partial femur resection plus ipsilateral iliac $\mathrm{LN}$ dissection, and adjuvant radiotherapy on the tumor bed and on the right iliac region. A first multifocal intrabdominal/retroperitoneal relapse was detected in February 2008, and was treated with high-dose ifosfamide plus complete surgical resection. In December 2009, lung metastases appeared. A third-line chemotherapy with trabectedin was started, with progression. Lacking any conventional alternative, in June 2011 the patient started sunitinib $37.5 \mathrm{mg} /$ day, on a continuous dosing regimen. Treatment was provided within a compassionate use program, with the approval of the Institutional Ethics Committee. At that time the disease involved the lungs, liver, abdomen, soft tissues, as confirmed by computed tomography scan (CT) and [18F]fluorodeoxyglucosepositron emission tomography scan (PET). No evidence of relapse to the primary tumor site was observed. Patient was asymptomatic, ECOG performance status (PS) $=1$.

A complete response by PET to all lesions was evident after 4 weeks of treatment (Figure 2B) with a marked decrease in maximum SUV, along with a decrease in tumor size and contrast uptake on CT (Figure 3B).

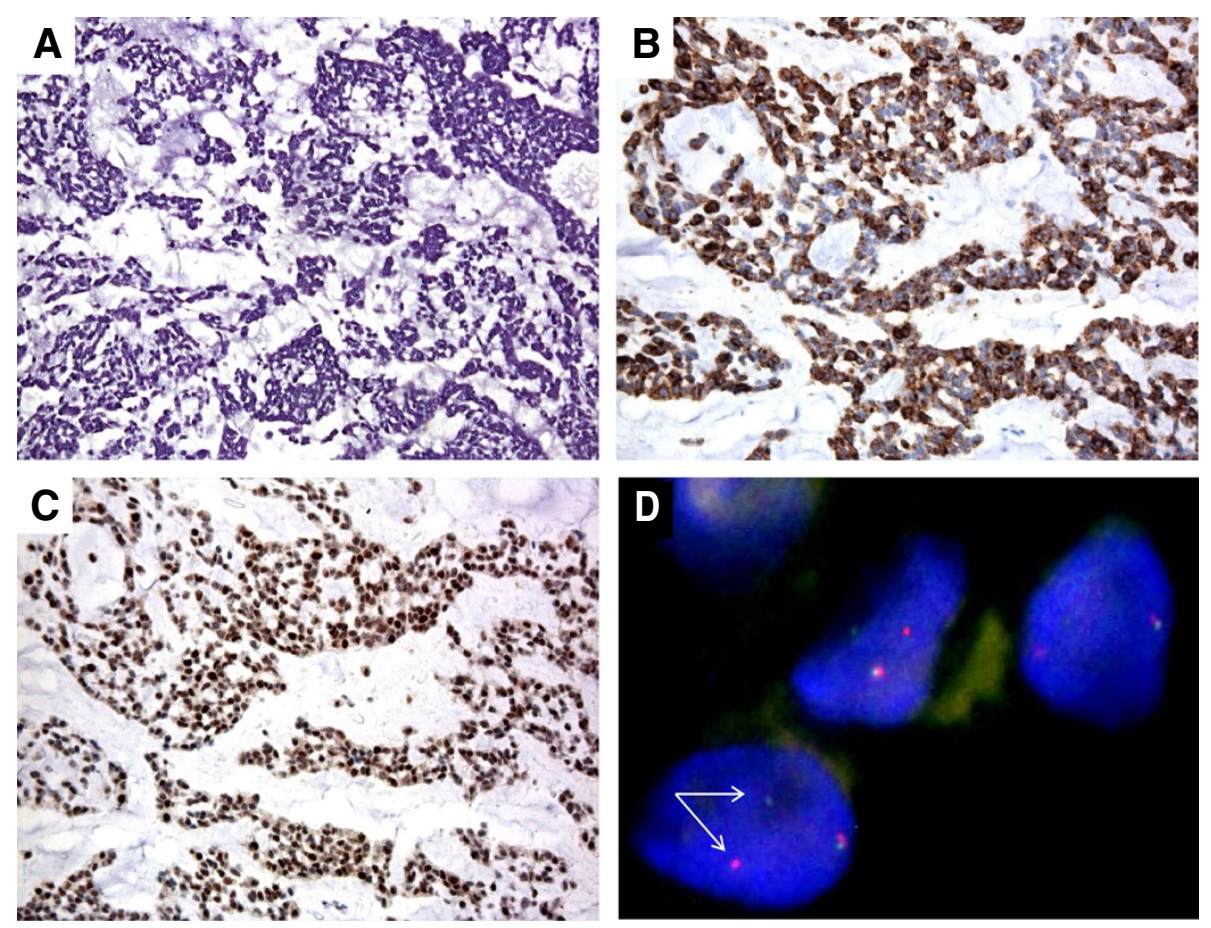

Figure 1 Patient 1 tumor biopsy consistent with the diagnosis of extraskeletal myxoid chondrosarcoma. Histology shows nests/cords of epithelioid cells embedded into a myxoid matrix (HandE) (panel A), characterized by diffuse pancytokeratin (AE1/AE3) cytoplasmic decoration (panel B) and PPARG nuclear expression (panel C). Panel D shows the FISH rearranged pattern for CHN, consisting in a fusion green/red signal, corresponding to an intact copy of the gene, and a split signal (red and green separated, white arrows) corresponding to the rearranged allele. 


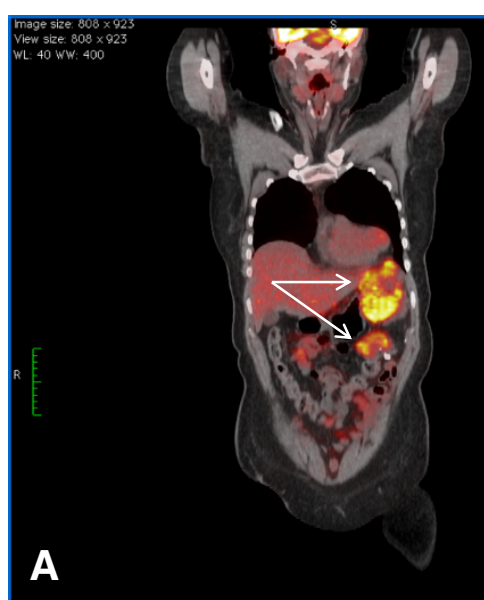

baseline

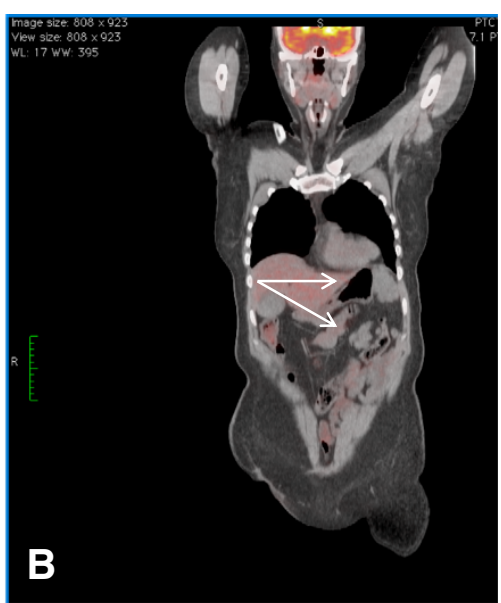

+1 mos

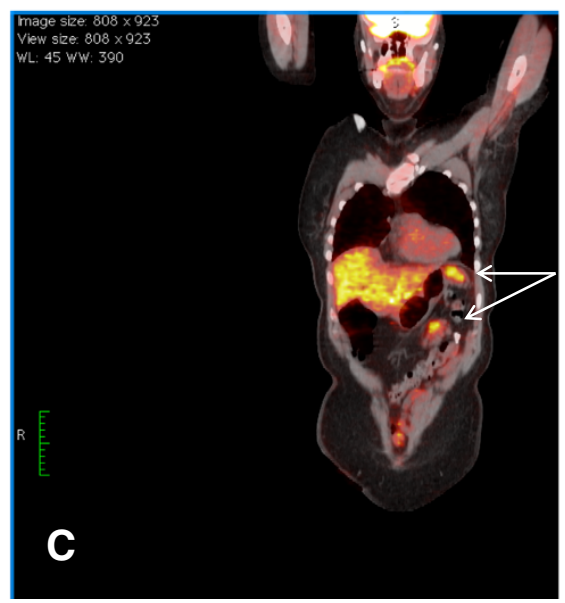

+6 mos

Figure 2 [18F]fluorodeoxyglucose-positron emission tomography scan (PET) tumor assessment. In panels $\mathbf{A}$ and $\mathbf{B}$ (white arrows) PET shows a marked decrease in maximum SUV comparing the tumor before and after 4 weeks of treatment. Panel $\mathbf{C}$ shows an interval tumor progression at 6 months from baseline, i.e. after 2 months from sunitinib interruption and treatment with antibiotics.

Unfortunately, 4 weeks after starting treatment, therapy was complicated by an abscess to the primary site, around femoral fixation. The infection was confirmed by clinical signs (fever, leukocytosis, redness, calor and pain located to the thigh), CT and PET. In fact, CT showed a tumor response to all sites, as described above, together with a new mass in the right gluteus muscles characterized by fluid content with rim enhancement (Figure 4C) which was not evident at baseline (Figure 4A). It was located below the painful right tight skin area. Consistently, that area became positive on PET (Figure 4D). Sunitinib was thus interrupted after 5 weeks and antibiotics were started, with progressive resolution of the infection, as confirmed by CT and PET three months later (Figure 4F). At that time, CT confirmed the tumor response to sunitinib with evidence of $>30 \%$ shrinkage, thus consistent with a RECIST response (Figure 3C). The patient was not on treatment anymore, due to the complicated tumor response. A new progression was observed 5 months after treatment interruption (6 months from baseline), as shown by PET and CT in Figure $2 \mathrm{C}$ and Figure 3D. Thus sunitinib $37.5 \mathrm{mg} /$ day was re-established, achieving a new $\mathrm{CT}$ response one month later (Figure 3E).

Following this experience another 67-years old consecutive patients, ECOG PS 1, affected by a EWSR1-CHN translocated progressive EMCS, metastatic to the lung and LN, pretreated with multiple lines of chemotherapy, started sunitinib in October 2011. After 3 months of treatment with sunitinib $37.5 \mathrm{mg} /$ day a tumor disease stabilization was observed. At that point sunitinib was tentatively increased to $50 \mathrm{~g} /$ day, with a dimensional response by CT detectable after 3 months of therapy, 6 months from baseline. 


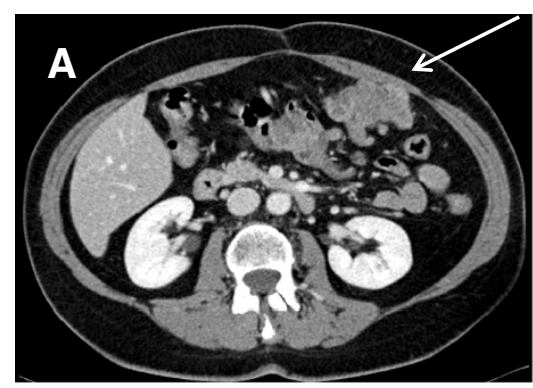

baseline
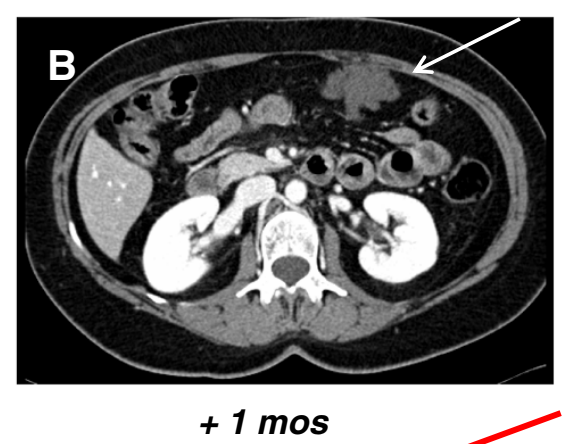

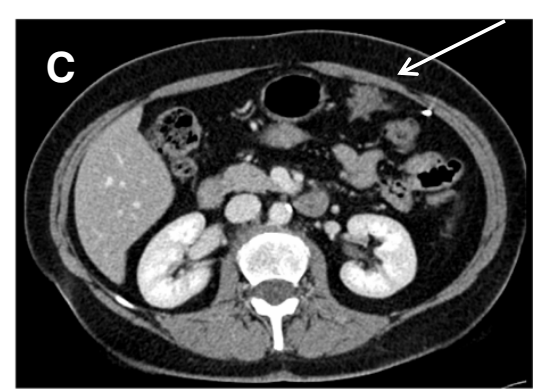

+4 mos

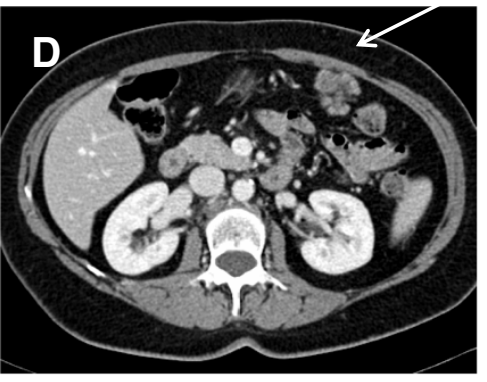

+6 mos

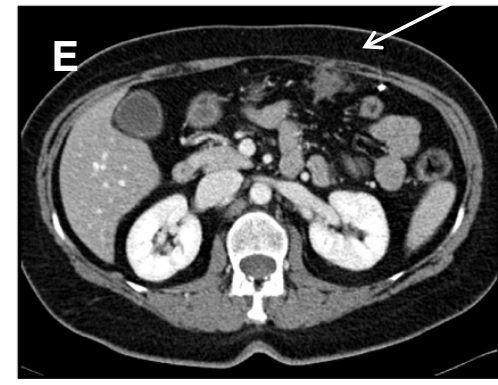

+7 mos

Figure 3 Computed Tomography scan (CT) tumor assessment. In panels A, B, C (white arrows) CT, venous phase, after contrast medium, shows a peritoneal metastasis at baseline, after 1 and 4 months of sunitinib with evidence of $30 \%$ decrease in tumor size and contrast uptake. Panel $\mathbf{D}$ shows an interval tumor progression at 6 months from baseline, i.e. after 2 months from sunitinib interruption, while panel $\mathbf{E}$ shows a new response at 7 months, 4 weeks after restoring sunitinib.

Both patients are still on treatment and responsive, 11 and 8 months from baseline, respectively, without evidence of further major side effects.

\section{Discussion}

We report on the tumor response to sunitinib in two consecutive patients with pretreated, progressive, metastatic extraskeletal myxoid chondrosarcoma, carrying the EWSR1-CHN translocation. In the first case the response was evident after only one month of treatment and marked by shrinkage of all lesions, on the face of a complete PET response. Decrease in tumor size was confirmed by further CT scans for 4 months after stopping treatment because of a therapy-related abscess. When disease progressed after 4 months from sunitinib discontinuation, response was restored by restarting treatment. At 9+ months of follow-up, tumor response was confirmed. In the second patient a dimensional response was evident at 6 months, after an initial tumor stabilization.

EMCS is a very rare STS, with low sensitivity to cytotoxic chemotherapy $[15,20-24]$. The only responses to chemotherapy were reported by McGrory in 2 of 6 metastatic EMCS patients responsive to a multi-agents chemotherapy [21], and in one patient treated with anthracycline plus ifosfamide described by Han [24]. No objective responses were observed in the MD Andersen series [23] of 10 patients receiving doxorubicin and dacarbazine-based regimens, nor in the series of 21 patients treated with different regimens, mostly anthracycline-based, reported by Memorial Sloan Kettering and Royal Marsden [20]. Finally, a response to interferon- $\alpha-2 b$ was also described [27]. To our knowledge, this is the first report on the activity of an antiangiogenic agent in EMCS.

Sunitinib is a multi-targeted tyrosine-kinase (RTK) inhibitor and antiangiogenic drug approved for the treatment of gastrointestinal stromal tumor (GIST) and renal cancer [28,29]. Evidence of activity in selected STS subtypes other than GIST has been provided [30-34]. Responses are often non dimensional, with some exception as for alveolar soft part sarcoma (ASPS) [30]. Of interest, in these patients with ESMC we could observe a major dimensional response.

Unlike GIST, no specific genetic alterations associated with sensitivity to sunitinib have been identified in STS. As shown in ASPS [30], another STS bearing a translocation, the antitumor activity of sunitinib is unlikely to be directly linked to the fusion-protein. In the absence of selective targets and known mechanisms of action, 

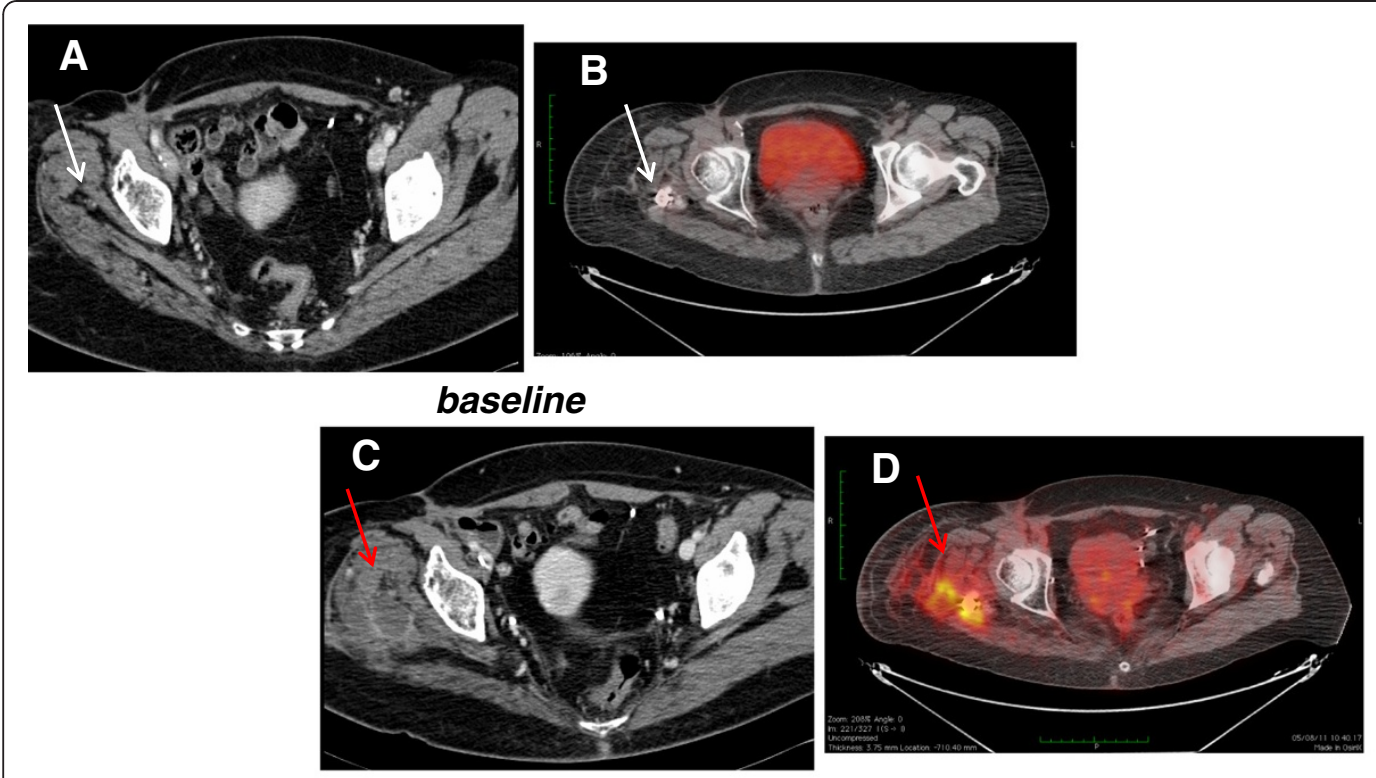

\section{+1 mos}

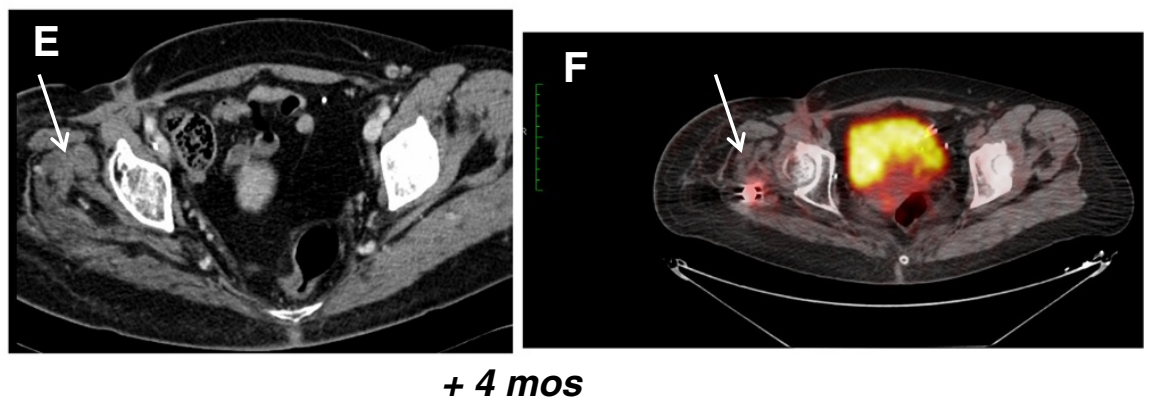

Figure 4 Computed Tomography scan (CT) and [18F]fluorodeoxyglucose-positron emission tomography scan (PET) abscess evaluation. CT showed a new mass in the right gluteus muscles characterized by fluid content with rim enhancement (panel $\mathbf{C}$ ) which was not evident at baseline (panel A). That area became positive on PET (panel $\mathbf{D}$ ) compared to baseline (panel B). CT (panel E) and PET (panel F) confirmed the resolution of the infection, three months later after sunitinib interruption and treatment with antibiotics.

sunitinib antiangiogenic activity as well as an effect on the autocrine-paracrine PDGFR/VEGFR activation-loop have been advocated as possible explanations for its antitumor activity [30]. Even in EMCS the fusion-protein is unlikely to be related to sunitinib sensitivity [12]. Unfortunately, due to absence of untreated frozen material, we could not assess the RTK activation profile.

The most common toxicities with sunitinib are handfoot syndrome, rash, fatigue, hypertension, hypothyroidism and diarrhea. It is known that the sunitinib and other antiangiogenetic agents can interfere also with the normal vasculature formation and, possibly, with the T-cell mediated immunity $[28,35,36]$. This can lead to rare complications, such as abscess formation [37-39]. In our first patient, the abscess originated at a site where there was no evidence of disease and was probably related to the presence of the foreign material for the femoral fixation. For the differential diagnosis, CT is viewed as the most accurate exam. In our case the presence of clear clinical signs of infection, as fever, leukocytosis, calor and redness of the skin, were of much help to rule out an isolated disease progression. A conservative approach was enough to heal the abscess and a drainage could be avoided. However, CT findings may sometimes be insufficient for the diagnosis and a biopsy can be necessary to rule out a disease progression.

\section{Conclusion}

A tumor response to sunitinib was seen in two consecutive patients with EMCS. In one case the response was complicated by infection and was restored when treatment could be restarted after a while. Differential diagnosis of complicated tumor response versus tumor progression was crucial to continue with therapy in the first responding patient. Further prospective studies are needed to confirm these results and to better understand the molecular basis for the activity of sunitinib in this disease. 


\section{Consent}

Written informed consent was obtained from the patients for publication of this Case Report and any accompanying legend. A copy of the written informed consents are available for review by the Editor-in-Chief of this journal.

\section{Competing interests}

Stacchiotti S Pfizer srl: travel coverage for medical meetings, research funding. Casali PG Pfizer spa: advisory, research funding.

\section{Authors' contributions}

SS, PD, SP, AG and PGC contributed to the conception and design, to the analysis and interpretation of data, to manuscript drafting PD and TN carried out the molecular study CM carried out the radiological evaluation and contributed to the analysis and interpretation of data AR contributed case material, and contributed to the analysis and interpretation of data All the authors read and approved the final manuscript

\section{Author details}

${ }^{1}$ Department of Cancer Medicine, Adult Sarcoma Medical Oncology Unit, Fondazione IRCCS Istituto Nazionale Tumori Milan, via Venezian 1, 20133, Milan, Italy. ${ }^{2}$ Department of Pathology and Laboratory of Molecular Pathology, Fondazione IRCCS Istituto Nazionale Tumori Milan, Milan, Italy. ${ }^{3}$ Department of Radiology, Fondazione IRCCS Istituto Nazionale Tumori Milan, Milan, Italy. ${ }^{4}$ Oncology Department, University Hospital Santa Chiara, Pisa, Italy. ${ }^{5}$ Department of Surgery, Fondazione IRCCS Istituto Nazionale Tumori Milan, Milan, Italy.

Received: 17 August 2012 Accepted: 30 September 2012 Published: 11 October 2012

\section{References}

1. Enzinger M, Shiraki M: Extraskeletal myxoid chondrosarcoma: an analysis of 34 cases. Hum Pathol 1972, 3:421-435.

2. Fletcher CDM, Unni KK, Mertens F (Eds): Tumours of Soft tissue and Bone. Pathology and Genetics. World Health Organization Classification of Tumours. Lyon: IARC Press; 2002

3. Aigner T, Oliveira AM, Nascimento AG: Extraskeletal myxoid chondrosarcomas do not show a chondrocytic phenotype. Mod Pathol 2004, 17:214-221.

4. Panagopoulos I, Mertens F, Isaksson M, et al: Molecular genetic characterization of the EWS/CHN and RBP56/CHN fusion genes in extraskeletal myxoid chondrosarcoma. Genes Chromosomes Cancer 2002, 35:340-352.

5. Subramanian S, West RB, Marinelli RJ, et al: The gene expression profile of extraskeletal myxoid chondrosarcoma. J Pathol 2005, 206:433-444.

6. Goh YW, Spagnolo DV, Platten M, et al: Extraskeletal myxoid chondrosarcoma: a light microscopic, immunohistochemical, ultrastructural and immuno-ultrastructural study indicating neuroendocrine differentiation. Histopathology 2001, 39:514-524

7. Hirabayashi Y, Ishida T, Yoshida MA, et al: Translocation (9;22)(q22;q12). A recurrent chromosome abnormality in extraskeletal myxoid chondrosarcoma. Cancer Genet Cytogenet 1995, 81:33-37.

8. Stenman G, Andersson H, Mandahl N, Meis-Kindblom JM, Kindblom LG: Translocation $\mathrm{t}(9 ; 22)(\mathrm{q} 22 ; \mathrm{q} 12)$ is a primary cytogenetic abnormality in extraskeletal myxoid chondrosarcoma. Int J Cancer 1995, 62:398-402.

9. Sjögren H, Meis-Kindblom J, Kindblom LG, et al: Fusion of the EWS-related gene TAF2N to TEC in extraskeletal myxoid chondrosarcoma. Cancer Res 1999, 59:5064-5067.

10. Sjögren H, Wedell B, Meis-Kindblom JM, Kindblom LG, Stenman G: Fusion of the NH2-terminal domain of the basic helix-loop-helix protein TCF12 to TEC in extraskeletal myxoid chondrosarcoma with translocation $t$ (9;15)(q22;q21). Cancer Res. 2000 Dec 15;60(24):6832-5. Erratum in. Cancer Res 2001, 61:2339.

11. Filion C, Labelle $Y$ : The oncogenic fusion protein EWS/NOR-1 induces transformation of CFK2 chondrogenic cells. Exp Cell Res 2004, 297:585-592.
12. Filion C, Motoi T, Olshen AB, et al: The EWSR1/NR4A3 fusion protein of extraskeletal myxoid chondrosarcoma activates the PPARG nuclear receptor gene. J Pathol 2009, 217:83-93.

13. Lucas DR, Fletcher CD, Adsay NV, et al: High-grade extraskeletal myxoid chondrosarcoma: a high-grade epithelioid malignancy. Histopathology 1999, 35:201-208.

14. Antonescu CR, Argani P, Erlandson RA, Healey JH, et al: Skeletal and extraskeletal myxoid chondrosarcoma: a comparative clinicopathologic, ultrastructural, and molecular study. Cancer 1998, 83:1504-1521.

15. Saleh G, Evans HL, Ro JY, et al: Extraskeletal myxoid chondrosarcoma. A clinicopathologic study of ten patients with long-term follow-up. Cancer 1992, 70:2827-2830.

16. Meis-Kindblom JM, Bergh P, Gunterberg B, Kindblom LG: Extraskeletal myxoid chondrosarcoma: a reappraisal of its morphologic spectrum and prognostic factors based on 117 cases. Am J Surg Pathol 1999, 23:636-650.

17. Ogura K, Fujiwara T, Beppu Y, et al: Extraskeletal myxoid chondrosarcoma: a review of 23 patients treated at a single referral center with long-term follow-up. Arch Ortop Thauma Surg 2012, 132:1379-1386.

18. Okamoto S, Hisaoka M, Ishida T, et al: Extraskeletal myxoid chondrosarcoma: a clinicopathologic, immunohistochemical, and molecular analysis of 18 cases. Hum Pathol 2001, 32:1116-1124.

19. Oliveira AM, Sebo TJ, McGrory JE, et al: Extraskeletal myxoid chondrosarcoma: a clinicopathologic, immunohistochemical, and ploidy analysis of 23 cases. Mod Pathol 2000, 13:900-908.

20. Drilon AD, Popat S, Bhuchar G, et al: Extraskeletal Myxoid Chondrosarcoma: A Retrospective Review From 2 Referral Centers Emphasizing Long-term Outcomes With Surgery and Chemotherapy. Cancer 2008, 113:3364-3371.

21. McGrory JE, Rock MG, Nascimento AG, et al: Extraskeletal myxoid chondrosarcoma. Clin Orthop Relat Res 2001, 382:185-190.

22. Kawaguchi S, Wada T, Nagoya S, et al: Extraskeletal myxoid chondrosarcoma: a multi-institutional study of 42 cases in Japan. Cancer 2003, 97:1285-1292.

23. Patel SR, Burgess MA, Papadopoulos NE, et al: Extraskeletal myxoid chondrosarcoma. Long-term experience with chemotherapy. Am J Clin Oncol 1995, 18:161-163.

24. Han K, Sun YJ, Shen Z, et al: Extraskeletal myxoid chondrosarcoma: a case report of complete remission by chemotherapy and review of the literature. BMJ Case Rep 2010, pii(bcr07):2009-2128.

25. Hornick JL, Fletcher CD: Myoepithelial tumors of soft tissue: a clinicopathologic and immunohistochemical study of 101 cases with evaluation of prognostic parameters. Am J Surg Pathol 2003, 27:1183-1196

26. Antonescu CR, Zhang L, Chang NE, et al: EWSR1-POU5F1 fusion in soft tissue myoepithelial tumors. A molecular analysis of sixty-six cases, including soft tissue, bone, and visceral lesions, showing common involvement of the EWSR1 gene. Genes Chromosomes Cancer 2010, 49:1114-1124

27. Rubinger $M$, Plenderleithi $H$, Lertzman $M$, et al: Metastatic extraskeletal myxoid chondrosarcoma. Successful therapy with interferon alfa-2b. Chest 1995, 108:281-282.

28. Demetri GD, Van Oosterom AT, Garrett CR, et al: Efficacy and safety of sunitinib in patients with advanced gastrointestinal stromal tumour after failure of imatinib: a randomised controlled trial. Lancet 2006, 368:1329-1338.

29. Motzer RJ, Rini BI, Bukowski RM, et al: Sunitinib in patients with metastatic renal cell carcinoma. JAMA 2006, 295:2516-2524.

30. Stacchiotti S, Tamborini E, Marrari A, Brich S, Rota SA, Orsenigo M, Crippa F, Morosi C, Gronchi A, Pierotti MA, Casali PG, Pilotti S: Response to Sunitinib Malate in Advanced Alveolar Soft Part Sarcoma (ASPS). Clin Cancer Res 2009, 15:1096-1104.

31. Stacchiotti $S$, Negri T, Libertini $M$, et al: Sunitinib in solitary fibrous tumor. Ann Oncol 2012,: in press.

32. George S, Merriam P, Maki RG, et al: Multicenter phase II trial of sunitinib in the treatment of non-gastrointestinal stromal tumor sarcomas. J Clin Oncol 2009, 27:3154-3160.

33. Domont J, Massard C, Lassau N, et al: Hemangiopericytoma and antiangiogenic therapy: clinical benefit of antiangiogenic therapy (sorafenib and sunitinib) in relapsed Malignant Haemangioperyctoma/ Solitary Fibrous Tumour. Invest New Drugs 2010, 28:199-202. 
34. Stacchiotti S, Grosso F, Negri T, Palassini E, Morosi C, Pilotti S, Gronchi A, Casali PG: Tumor response to sunitinib malate observed in clear cell sarcoma. Ann Oncol 2010, 21:1130-1131.

35. Desai J, Yassa L, Marqusee E, et al: Hypothyroidism after sunitinib treatment for patients with gastrointestinal stromal tumors. Ann Intern Med 2006, 145:660-664.

36. Motzer RJ, Hutson TE, Olsen MR, et al: Randomized Phase II Trial of sunitinib on an intermittent versus continuous dosing schedule as first-line therapy for advanced renal cell carcinoma. J Clin Oncol 2012, 12:1371-1377.

37. Michalaki V, Arkadopoulos N, Kondi-Pafiti A, et al: Abscess formation mimicking disease progression, in a patient with metastatic renal cell carcinoma during sunitinib treatment. World I Surg Oncol 2010, 8:45.

38. Dembry LM: Renal and perirenal abscesses. Curr Treat Options Infect Dis 2002, 4:21-30

39. Geeting GK, Shaikh N: Renal abscess. J Emerg Med 2006, 31:99-100.

doi:10.1186/2045-3329-2-22

Cite this article as: Stacchiotti et al.: Extraskeletal myxoid

chondrosarcoma: tumor response to sunitinib. Clinical Sarcoma Research 2012 2:22.

\section{Submit your next manuscript to BioMed Central and take full advantage of:}

- Convenient online submission

- Thorough peer review

- No space constraints or color figure charges

- Immediate publication on acceptance

- Inclusion in PubMed, CAS, Scopus and Google Scholar

- Research which is freely available for redistribution 